Chirurg 2018 $\cdot 89: 66$

https://doi.org/10.1007/s00104-017-0565-2

Online publiziert: 30 . November 2017

(c) Springer Medizin Verlag $\mathrm{GmbH}$, ein Teil von Springer Nature 2017

CrossMark

W. Schröder · C. J. Bruns

Klinik für Allgemein-, Viszeral- und Tumorchirurgie, Universitätsklinik Köln, Köln, Deutschland

\title{
Chirurgische Strategien beim Kardiakarzinom (AEG Typ II)
}

pe vergleichbar niedrig (TAE $85,9 \%$ vs. THG $83,9 \%, p=0,719)$. Die mediane Anzahl der resezierten Lymphknoten lag in beiden Gruppen bei 24. Das mediane Gesamtüberleben lag bei 38,4 Monaten, das 5-Jahres-Überleben bei 42,8\%. Die 3- und 5-Jahres-Überlebensraten waren in der THG-Gruppe signifikant geringer als bei Patienten mit TAE (48,5\% vs. $69,6 \%$ und $38,8 \%$ vs. $57,5 \%, p=$ 0,02). In der multivariaten Analyse war das chirurgische Verfahren ein unabhängiger Prognosefaktor für das Überleben.

Fazit. Nach Analyse dieses bisher größten Kollektivs zu AEG-Typ-II-Karzinomen favorisieren die Autoren aufgrund der besseren Überlebensdaten die transthorakale Ösophagektomie. Wie aber in den bisher publizierten retrospektiven Untersuchungen bleibt auch hier eine sichere Zuordnung zum AEG-Typ II schwierig. Im methodischen Teil ist nicht hinreichend definiert, ob bei den neoadjuvant therapierten Patienten die Zuordnung aufgrund der Endoskopie vor oder nach Chemotherapie erfolgte und der endoskopische Befund mit der makropathologischen Beurteilung des Resektates insbesondere bei Patienten mit „major“ oder „complete response“ korrelierte. Ein weiterer Bias dieser Arbeit ist die unterschiedliche Altersstruktur der beiden untersuchten Gruppen. Deshalb bleibt die Kernfrage nach dem onkologisch adäquaten Resektionsausmaß für AEG-Typ-II-Karzinome weiterhin unbeantwortet und kann wie von den Autoren der Arbeit auch gefolgert nur durch ein prospektiv-randomisiertes Studiendesign geklärt werden.

\section{Korrespondenzadresse}

Prof. Dr. W. Schröder

Klinik für Allgemein-, Viszeral- und

Tumorchirurgie, Universitätsklinik Köln

Kerpener Str. 62, 50937 Köln, Deutschland

wolfgang.schroeder@uni-koeln.de

Interessenkonflikt. W.Schröder und C.J.Bruns geben an, dass kein Interessenkonflikt besteht. 